\title{
Development and characterization of a PDMS-based masking method for microfabricated Oral drug delivery devices
}

Kamguyan, Khorshid; Thamdrup, Lasse Højlund Eklund; Vaut, Lukas; Nielsen, Line Hagner; Zor, Kinga; Boisen, Anja

Published in:

Biomedical Microdevices

Link to article, DOI:

$10.1007 / \mathrm{s} 10544-020-00490-8$

Publication date:

2020

Document Version

Peer reviewed version

Link back to DTU Orbit

Citation $(A P A)$ :

Kamguyan, K., Thamdrup, L. H. E., Vaut, L., Nielsen, L. H., Zor, K., \& Boisen, A. (2020). Development and characterization of a PDMS-based masking method for microfabricated Oral drug delivery devices. Biomedical Microdevices, 22(2), [35]. https://doi.org/10.1007/s10544-020-00490-8

\section{General rights}

Copyright and moral rights for the publications made accessible in the public portal are retained by the authors and/or other copyright owners and it is a condition of accessing publications that users recognise and abide by the legal requirements associated with these rights.

- Users may download and print one copy of any publication from the public portal for the purpose of private study or research.

- You may not further distribute the material or use it for any profit-making activity or commercial gain

- You may freely distribute the URL identifying the publication in the public portal 


\title{
Development and Characterization of a PDMS-based Masking Method for
} Microfabricated Oral Drug Delivery Devices

Khorshid Kamguyan ${ }^{1}$, Lasse Højlund Eklund Thamdrup ${ }^{1}$, Lukas Vaut ${ }^{1}$, Line Hagner Nielsen ${ }^{1}$, Kinga Zor $^{1}$, Anja Boisen ${ }^{1}$

\begin{abstract}
Acknowledgements The authors would like to acknowledge the Novo Nordisk Foundation (NNF17OC0026910) for funding the project MIMIO - Microstructures, microbiota and oral delivery and Danmarks Grundforskningsfond og Villum Fondens Center for Intelligent Drug Delivery and Sensing Using Microcontainers and Nanomechanics (IDUN) whose research is funded by the Danish National Research Foundation (DNRF122) and Villum Fonden (Grant No. 9301).

The authors would like to thank Zarmeena Abid from National Center for Nanofabrication and Characterization, Technical University of Denmark for supplying the PCL microcontainers for this project and Jesper Scheel from the Department of Health Technology, Technical University of Denmark for photography of the PDMS mask fabrication process.
\end{abstract}

\footnotetext{
${ }^{1}$ The Danish National Research Foundation and Villum Foundation's Center for Intelligent Drug Delivery and Sensing Using Microcontainers and Nanomechanics, Department of Health Technology, Technical University of Denmark, Kgs. Lyngby, 2800, Denmark
}

Correspondence to: Khorshid Kamguyan; E-mail: khokam@dtu.dk; Orcid ID: 0000-0003-1754-3434 
Abstract With the growing popularity and application of microfabricated devices in oral drug delivery (ODD), masking technologies for drug loading and surface modification become highly relevant. Considering the speed of design and fabrication processes and the necessity for continuous iterations of e.g. the shape and sizes of the devices during the optimization process, there is a need for adaptable, precise and low-cost masking techniques. Here, a novel method is presented for masking ODD microdevices, namely microcontainers, using the physical characteristics of polydimethylsiloxane (PDMS). When compared to a rigid microfabricated shadow mask, used for filling drugs in microcontainers, the PDMS masking technique allows more facile and precise loading of higher quantities of an active compound, without the need of alignment. The method provides flexibility and is adjustable to devices fabricated from different materials with various geometries, topologies and sizes. This userfriendly flexible masking method overcomes the limitations of other masking techniques and is certainly not limited to ODD and is recommended for a wide range of microdevices.

Keywords Microfabricated devices, Oral drug delivery, Flexible masks, Polydimethylsiloxane, Microcontainers

\section{Introduction}

Microfabrication technologies are commonly used for sensor manufacturing(V. K. Varadan 2006), surface partterning(Jiang et al. 2011) and production of microfluidic systems(Silverio and Cardoso de Freitas 2018). With the development of new technologies, utilizing microfabricted devices becomes feasible and increasingly relevant in various research areas, including oral drug delivery (ODD)(Nielsen et al. 2018; Zhang et al. 2017). Purposefully designed and microfabricated drug delivery systems can address universal challenges in ODD, such as protection of an active compound(Nielsen et al. 2015), improved absorption of peptides(Jørgensen et al. 2019) and small molecules(Nielsen et al. 2016), bioadhesion(Fox et al. 2016) and controlled kinetics of drug release(Chirra and Desai 2012). As these devices become smaller and more complex, the methods for their handling and manipulation, e.g. drug loading(Fox et al. 2015) or surface chemical modification(Srivastava et al. 2019) need to be more precise and controllable. Alteration of surface properties, loading of drug compounds and general manipulation of microdevices can be achieved by masking techniques. Masking gives selective surface access through coverage of unwanted areas and exposure of a desired portion of a substrate(Luo et al. 1998; Wu et al. 1993; Courcimault and Allen 2004).

Conventionally, shadow masks are fabricated from tungsten, tantalum, silicon or metals, e.g. stainless steel using electrical discharge machining(Schallenberg et al. 2002; Becker and Gärtner 2008) or electroplating of Ni(Keller et al. 2013). However, as more complex microdevices are designed to address physiological challenges, e.g. mucoadhesion(Tao and Desai 2005), microfabrication of rigid masks becomes costly, time-consuming and inefficient. Thus, the need for flexible and facile masking methods has rapidly grown, giving rise to fabrication of shadow masks made from flexible elastomers, mainly parylene and polydimethylsiloxane (PDMS)(Selvarasah et al. 2008; Chen et al. 2014). PDMS is the most commonly used polymer in microfabrication technologies for producing microelectromechanical systems(Schneider et al. 2009) and microfluidics devices(Roh et al. 2016), through procedures like soft lithography and microscale casting(Silverio and Cardoso de Freitas 2018). This highly processable polymer has also been utilized for development and fabrication of biomedical devices in drug delivery(Chen et al. 2009; Chung et al.). Low cost, biocompatibility, chemical inertness, ease of manipulation and mechanical flexibility give this elastomer a unique position in the development of microdevices and fast prototyping technologies(Kimura et al. 2019). The low surface energy and unique rheological behavior of PDMS result in a steady flow of the resin at room temperature, thus allowing precise area coverage on a small scale(Ghannam and Esmail 1998; Sowers et al. 2016). These properties enable the use of PDMS for masking complex microdevices attached to a substrate(Roh et al. 2016). Sayah et al. utilized a PDMS-based technique for masking micro-sized structures for sensing applications, by pouring the resin onto the top of the devices and scraping off the excess material from the top surface(2005). This approach can be invasive and harmful to microstructures and requires post-processing and clean-up steps. More importantly, in the case of complex devices, e.g. microreservoir-based devices or topology optimized microdevices, this method is no longer applicable. More recently, Chen et al. proposed using PDMS for masking of dimple-shaped microstructures, used for modification of surface properties, using parallel discs and isolating the area in between the 3D structures. The PDMS resin was then pumped inside the isolated space leaving the top of the micro-dimples, 2 
uncoated(Chen et al. 2015). Although, this method is more efficient and less invasive than the former techniques, it is also not applicable in the case of microdevices with complex geometries or enhanced roughness of the top surface.

Here, we present a novel easy-to-use method for masking the areas in between microfabricated devices attached to a support substrate, using PDMS. For demonstrating the method, we have used ODD microfabricated devices, namely microcontainers coined by Nielsen et al(Nielsen et al. 2012). Microcontainers are polymeric cylindrical microdevices with a hollow reservoir on one side, which are designed to increase oral bioavailability and protection of drugs. The microcontainers are fabricated on Si wafer substrates for ease of handling and manipulation. After fabrication, the reservoirs are loaded with an active compound then sealed with a polymeric lid, after which the devices can be detached from the sacrificial support substrate underneath, leaving them ready for testing e.g. in vivo(Mazzoni et al. 2017). The drug loading method e.g. inkjet printing(Fox et al. 2017), powder embossing(Abid et al. 2017) or supercritical impregnation(Marizza et al. 2016) is chosen based on the physical properties of the active compound. One of the methods for loading drugs in powder form in the microcontainers involves the use of a microfabricated shadow mask in order to assure precise filling in the reservoirs and not in the area between the microdevices(Abid et al. 2017). However, development of new designs in the optimization processes, e.g. changes in dimension and overall shape of the microdevices can be costly and time-consuming, as a new mask needs to be fabricated for each design. Moreover, application of such masks requires manual alignment on top of the devices, which can be slow and tedious, thus a self-aligning mask is highly desirable. In the following, fabrication of PDMS masks for microcontainers is described followed by characterization of the thermal, physical and mechanical behavior of the masks. Furthermore, the presented method is compared to the conventionally used Ni shadow masks for drug loading in microcontainers. Lastly, compatibility of the proposed masking method with changes in size, geometry, topology and material of the microdevices is evaluated in order to investigate how this method can overcome the limitations of previous masking technologies.

\section{Materials and Methods}

\subsection{Materials}

Sylgard ${ }^{\circledR} 184$ two part Silicone Elastomer (viscosity $($ mixed) $=3500 \mathrm{cP}$, specific gravity (cured) $=1.03$ ) containing the prepolymer base and the curing agent was obtained from Dow Corning (MI, USA) and Silicon (Si) wafers, 4inch (100) single-side polished, were obtained from Topsil GlobalWafers A/S (Frederikssund, Denmark). SU-8 2035, 2075 and SU-8 developer were purchased from micro resist technology GmbH (Berlin, Germany). Poly- $\varepsilon-$ caprolactone (PCL) $\left(\mathrm{Mn}=80,000 \mathrm{~g} \mathrm{~mol}^{-1}\right.$ ) was purchased from Sigma Aldrich (MO, USA), and HTM 140M V2 3D printing photopolymer from EnvisionTEC GmbH (Gladbeck, Germany). 5-Aminosalicylic acid (5-ASA) and paracetamol were supplied by Sigma Aldrich and indomethacin from Tokyo Chemical Industry (Tokyo, Japan). Phosphate buffered saline (PBS) was obtained from Sigma Aldrich.

\subsection{Fabrication of microdevices}

Cylindrical SU-8 microcontainers, microcontainers with micro-pillars on the sidewalls and microdevices having round, square and triangular geometries with outer diameters/sidelengths ranging from approximately $100 \mu \mathrm{m}$ to $370 \mu \mathrm{m}$ (Figure S3) were fabricated by UV lithography on Si substrates, based on a previously described fabrication scheme(Nielsen et al. 2012). Fabrication of the devices is described in more detail in supplementary information section S2. After fabrication, the wafers were cut into squared chips using a dicing saw (DISCO, automatic dicing saw, DAD 321, Japan).

PCL microcontainers were fabricated by a previously published method through compression molding(Abid et al. 2019). Ni stamps were hot embossed onto a PCL film with a thickness of $100 \mu \mathrm{m}$ using a Collin ${ }^{\circledR}$ Press, 300 SV (Collin Lab and Pilot Solutions GmbH, Maitenbeth, Germany). 
3D printed microcontainers were designed using OpenSCAD open source software and SolidWorks 2015 (Dassault Systèmes SolidWorks Corporation, USA) and fabricated by an EnvisionTec Micro Plus High-Res DLP 3D printer (EnvisionTEC GmbH, Gladbeck, Germany) according to a procedure previously reported(Vaut et al. 2019). After completion of the process, the 3D printed microdevices were sonicated for $5 \mathrm{~min}$ in isopropanol followed by a UV post-curing for $10 \mathrm{~min}$.

All microdevices were characterized using a TM3030Plus tabletop scanning electron microscope (SEM, Hitachi High Technologies Europe GmbH, Krefeld, Germany). Topology characterization on the microdevices was performed using conventional bright-field microscopy (Nikon Eclipse L200, Nikon Metrology, Tokyo, Japan) and vertical scanning interferometry (PLu Neox 3D Optical Profiler, Sensofar Metrology, Barcelona, Spain). Characterization of the devices is further described in supplementary information section S2.

\subsection{Fabrication of PDMS masks}

Sylgard 184 two parts were mixed manually with a 10:1 w/w ratio and degassed for 15 min to remove trapped air bubbles formed due to the mixing process. Si chips containing microcontainers were placed in chip trays (Entegris, MA, USA) with a single chip per well, as shown in Figure1-B1. $150 \mu 1$ of the resin mixture was initially poured onto each chip tray well, in the gap between the chip and the well walls, after which the area between the microcontainers on the chip was slowly filled. After $30 \pm 5 \mathrm{sec}$, another $150 \mu \mathrm{l}$ was added to each well to ensure complete wetting of the wanted surfaces. The flow was maintained by keeping the amount of liquid between the chip and the well walls constant. After complete coverage of the chip, the chip tray was kept in air at $37^{\circ} \mathrm{C}$ overnight (unless specified otherwise) for curing. After application for e.g. drug loading, the mask was gently removed by pulling the edge of the crosslinked polymer with a tweezer and detaching it from the $\mathrm{Si}$ chip bearing the microcontainers.

\subsection{Characterization of the masking process}

Physical and mechanical characteristics of the PDMS masks during the fabrication process were examined using a TA.XTplus texture analyzer (Stable Micro Systems Ltd, Godalming, UK) equipped with a $500 \mathrm{~g}$ loadcell. After a contact time of $30 \mathrm{sec}$ between a 3D printed polylactic acid (PLA) cylindrical reference substrate with a contact surface area of $250 \mathrm{~mm}^{2}$ and a contact force of $10 \mathrm{~g}$; the probe was moved upwards at a speed of 0.01 $\mathrm{mm} . \mathrm{s}^{-1}$. The measurements were performed every 10 min during the preparation of the PDMS masks. For characterization of effect of temperature on the fabrication procedure of the masks, the Si chips bearing microcontainers were put in glass petri dishes and masks were fabricated at $37,50,70,90$ or $100^{\circ} \mathrm{C}$. The optimized time for preparation of the masks was measured based on the physical and mechanical characterizations.

\subsection{Characterization of the drug loading process}

For the purpose of loading drugs into the microcontainers, either a microfabricated Ni shadow mask (supplementary information section S1) or the PDMS mask were utilized. Microcontainers were loaded with 5ASA, paracetamol or indomethacin by manually pressing the drug powder on top of the masked microcontainer chips using a spatula, after which a soft brush was used to remove excess powder from the top surface. The amount of drug filled in the devices, was measured by weighing the chips containing microcontainers before and after the loading process.

Drug release studies were conducted using a $\mu$-Diss profiler (Pion Inc, MA, USA). After removal of the masks, the chips containing microcontainers loaded with indomethacin, 5-ASA and paracetamol were attached to magnetic stirrers, covered with $\mathrm{PBS}\left(\mathrm{pH}=7.4\right.$ at $37^{\circ} \mathrm{C}$ ) and set at a speed of $100 \mathrm{rpm}$. $\mathrm{UV}$ absorbance was measured at $310 \mathrm{~nm}$ for indomethacin and $260 \mathrm{~nm}$ for 5-ASA and paracetamol, using a UV probe with a path length of $5 \mathrm{~mm}$. For all the measurements, calibration curves were constructed using drug concentrations ranging from 0.005 to $0.15 \mathrm{mg} / \mathrm{ml}$.

\subsection{Data analysis}


All experiments were performed in triplicates and the obtained data was plotted as mean \pm standard deviation. Model fitting, data plotting and statistical analysis were conducted with OriginLab software (OriginLab, MA, USA). A nonlinear regression analysis using an exponential decay function was used for data curve fitting. The fitted model was statistically analyzed using adjusted R-Squared and a reduced Chi-squared test for investigating data variability and goodness of fit.

\section{Results and Discussion}

\subsection{Fabrication and characterization of PDMS masks}

The fabrication procedure of the PDMS masks is illustrated in Figure 1-A. As illustrated, a liquid mixture of prepolymer and crosslinking agent was poured onto the chip tray near the edge of the chip containing the microcontainers. The liquid polymer then flowed through the space between the microdevices due to the capillary forces and the interfacial tension between the resin and the walls of the microdevices. After the polymer was fully crosslinked, the flexible elastomeric film was easily peeled off from the Si chip bearing the microcontainers as a support substrate. The capillary-driven flow of pre-cured PDMS resin and peeling off of the post-crosslinking mask is shown in Figure 1 (B1 - B2). Based on the SEM images of empty microcontainers before and after masking with PDMS as well as the removed mask, it is evident that the method has not harmed the microcontainers due to the inert chemistry of PDMS and flexibility of the mask after crosslinking (Figure 1 $(\mathrm{C} 1$ - C3)). The exact dimensions of the microcontainers is further described in Figure S2. We can see from the SEM images that the PDMS resin has embedded the microcontainers without entering the reservoir, thus not contaminating the drug loading process. In addition, it can also be observed from the images of the peeled off mask (Figure 1-C3) that the resin has not been in contact with the top of the microcontainer walls as the diameter of the holes in the peeled off mask is the same as the outer diameter of the microcontainers. It is noticeable that the proposed masking method did not need further alignment steps between the mask and the microdevices on the substrate as it was a product of the flow of the resin between the devices.

To characterize and optimize the masking process, the effect of temperature on the preparation time of the mask was investigated (Figure 2). The fabrication time of the PDMS masks embedding microcontainers on a Si chip was studied at different temperatures ranging from $37-100^{\circ} \mathrm{C}$. As presented in Figure 2-A, curing time rapidly decreases by increasing the temperature, from about $20 \mathrm{~h}$ at $37^{\circ} \mathrm{C}$ to $30 \mathrm{~min}$ at $70^{\circ} \mathrm{C}$ and only about $5 \mathrm{~min}$ at $100^{\circ} \mathrm{C}$. Moreover, a non-linear model was fitted on the temperature dependency of the masking process as follows,

$t=t_{0}+\alpha\left(e^{-T / \beta}\right)$

where the time for mask preparation $(\mathrm{t})$ is a function of the temperature $(\mathrm{T})$ for masking of SU-8 microcontainers with $250 \mu \mathrm{m}$ height on a Si wafer, using a one-phase exponential decay function. Model parameters $\mathrm{t}_{0}, \alpha$ and $\beta$ derived by curve fitting and statistical analysis is shown in Figure 2-C. Figure 2-B shows the curve fitting on the measured data. We can see that the model can successfully predict the mask preparation time for different temperatures, which is in accordance with the statistical analysis of the fitting (Figure 2-C). Although, deviation from this model is expected in the case of changing different aspects of the microdevices (dimensions, substrate chemistry and material) or the grade of PDMS, the parameters for optimizing the masking process are shown to be predictable. This reliability and adaptability is crucial as the microdevices are constantly optimized and redesigned based on the needs of the field.(Tao and Desai 2003; Vaut et al. 2020)

Previously, Dorogin and Persson(2018) studied the adhesion of PDMS to a glass ball as a function of crosslink density in order to investigate the contact mechanics of the polymer during the curing process. In the present study, the optimized time for preparation of the mask was investigated by examining the adhesion of the sample to a reference substrate, monitoring the direct effect of crosslink density on adhesiveness of PDMS. As the 
mixture of prepolymer and crosslinker approach the pot life, the viscosity of the liquid mixture increases until reaching a solid state(Winter and Chambon 1986), at which time the crosslinking process is not completed, but the solid state of the elastic polymer would allow manipulation of the sample, e.g. peeling off the mask. To characterize this process during the curing of PDMS, the adhesion of PDMS to a reference substrate was studied by tensile force measurements at different time intervals during the fabrication of PDMS masks at $70^{\circ} \mathrm{C}$. Figure 3-A shows representative force curves, during which the reference substrate was in contact with the mask for 30 sec and then detached by a constant velocity, as can be seen more clearly in the inset of Figure 3-A. We observe that $10 \mathrm{~min}$ after the start of the experiment, there was a significant increase in the peak force, which represents the adhesion between PDMS and the reference substrate. This change in peak force can be related to the increase of viscosity due to the start of the formation of a 3D crosslinked network in the PDMS mixture(Ghannam and Esmail 1998). After $20 \mathrm{~min}$, a decrease in peak force and a broader range for the area under the curve can be seen, which represents the elongation of partially crosslinked PDMS resin attached to the substrate(Dorogin and Persson 2018). This change can be related to further crosslinking of the polymer network leading to higher viscosity and reaching a viscoelastic state. After $30 \mathrm{~min}$, a sudden drop in both peak force and the range of the area under the curve was seen, which did not change noticeably after this point of time in the curing process. At this point in the crosslinking process of the polymer, the PDMS reached a solid elastic state and showed little adhesion to the reference substrate(Zosel 1991). To understand this process further, the work of adhesion for different time points was calculated. Figure 3-B displays the work of adhesion at each time point at $70^{\circ} \mathrm{C}$, calculated by the area under the force-displacement curves. It is evident from the chart that as the crosslinking process started the adhesion of the PDMS to the reference substrate increased, at which point the mask could not be used. However, by completion of the masking process samples became more elastic decreasing adhesion and stickiness, thus allowing the mask to be used for loading the microcontainers and detachment of the mask without harming the microstructures or risk of contamination. It can also be observed, that after $30 \mathrm{~min}$, the work of adhesion remained unchanged, confirming the completion of the fabrication process. At this time, the PDMS became easily detachable from the surface, making it suitable for non-invasive masking of micro-sized structures or devices.

\subsection{Comparison with a Ni shadow mask}

To evaluate the efficiency of the here presented masking approach, the performance of the PDMS mask was compared with a microfabricated Ni shadow mask (Figure S1) for loading powder drugs into microcontainers. Figure 4 shows SEM images of microcontainers filled with indomethacin as a model drug using a PDMS (A1 A2) and a Ni mask (B1 - B2). We can clearly see that using the PDMS mask resulted in a more efficient and clean loading process, while using the nickel mask led to an uneven and incomplete loading of the indomethacin powder. It also seems that the use of the PDMS mask resulted in a much more compact loading of the microdevices. For this matter, the efficiency of the masks was examined by comparing the drug loading capacity of microcontainers using both approaches.

Three different model drugs, 5-ASA, indomethacin, and paracetamol were filled in the microcontainers using the $\mathrm{Ni}$ and the PDMS mask, and the amount of drug loaded was measured and compared (Figure 4-C1). We can observe a noticeable difference between the drug loading capacity of the two approaches. The general difference for the loading capacity of the three drugs is due to the different characteristics of the powders (small crystal flakes); however, for each drug, we can see a two to three-fold higher loading capacity in the case of the PDMS mask. The impact of the PDMS-based method on the loading capacity of microcontainers was further examined by comparing the release profiles of indomethacin from loaded microcontainers, using the two masking methods (Figure 4-C2). The low aqueous solubility of indomethacin results in a distinct difference in the release profile of the drug loaded using the Ni mask compared with using the PDMS masking method. As can be seen in Figure 4$\mathrm{C} 2$, there is an obvious difference between the drug release profiles of the two masking techniques. For the samples loaded with the Ni mask, a burst release can be observed, which could be explained by a loosely loaded powder. On the other hand, the release profile of the indomethacin loaded using the PDMS mask showed a slower release, which stems from the highly packed powder filled in the microcontainers. In comparison with the loading of 5-ASA and paracetamol using the two masking methods, although the difference between the two techniques is visible in the SEM images (Figure S4), the difference between the release profiles becomes less 
noticeable as the aqueous solubility of the compounds increase (supplementary information S3). Higher loading efficiency by compact filling of the drug can be related to the flexible nature of PDMS, which allowed more compression on the masked devices and resulted in a better alignment with the microcontainers.

\subsection{Versatility of the masking method}

To further demonstrate the versatility and flexibility of the PDMS masking method, previously applied for selfpropelling micromotors(Srivastava et al. 2019), we have explored microdevices designed and fabricated in various geometries, sizes, topologies and materials. It can be observed from the SEM images that the PDMS mask had fully embedded microdevices shaped as triangles and squares (Figure 5 (A1 - A2)). The inert mixture of resin and crosslinker forming the PDMS mask could successfully cover the entire surface of the area in between the microdevices regardless of the shape or the sharp edges of the structures. Figure 5 (B1 - B2) shows cylindrical microdevices with two different sizes embedded in PDMS. It can be noticed that microdevices of both large and small sizes were fully masked without the resin flowing inside the reservoirs. To further demonstrate the versatility of this method, we have explored microdevices in two other materials, namely PCL and an acrylate-based photo-polymer used for 3D printing (HTM 140M V2). PCL microcontainers were fabricated in a previous study as biodegradable microdevices for oral drug delivery(Abid et al. 2019). Figure 5$\mathrm{C} 1$ shows the PCL microcontainers masked with PDMS, which had fully covered the area around the reservoir up to the sharp top edges. 3D printing was recently used as an alternative approach for fast prototyping of different designs for microcontainers(Vaut et al. 2019). Figure 5-C2 presents 3D printed microdevices embedded in PDMS leaving the top edges and inside of the reservoir, uncoated. It is noticeable that despite the softer edges of these microdevices, the PDMS resin does not overflow or enter inside the reservoir. It is worth to mention, that as the mechanism for this method is controlled wetting, the surface chemistry of the support substrate should be assessed in the case of changing the materials i.e. this method might not be suitable for super hydrophilic/hydrophobic surfaces.

To further highlight differences between this masking method and previously presented approaches using flexible shadow masks, microdevices with pillar-shaped structures on the top surface were used (Figure 6-A). It has been shown that by changing the surface topography of ODD microdevices, their intestinal retention can be increased for better drug absorption in the mucosa of the small intestine(Martin and Grove 2001; Vaut et al. 2020). However, drug loading into such devices can be challenging with previous masking technologies. For example, the use of rigid metallic shadow masks can be invasive for the fine microstructures of the devices. Previously, Chen et al.(2015) fabricated a device that allows pumping the PDMS resin into the desired areas by placing two flat substrates on the top and bottom surface of the microdevices. However, in the case of devices with 3D structures on the surface, this method is not applicable due to the empty space between the surface structures. Here, we have fabricated microdevices with the same criteria and examined the PDMS mask with regard to the fragile 3D structures on the surface. Figure 6 (B - C) shows SEM images of these devices before and after removal of the PDMS mask. It can be observed that the PDMS resin not only covered the areas between the microdevices, but it also covered the empty areas between the pillars without overflowing into the reservoirs. In addition, after removal of the PDMS mask, the fragile pillars were intact and unaffected by the pulling force from the process of detaching the mask (Figure 6-C). Therefore, it was evident that the PDMS masking method proposed here, can be used for microdevices with complex designs and protect the integrity of fragile structures during processing.

\section{Conclusion}

A novel method for fabrication of flexible PDMS masks was demonstrated for surface manupilation of microconrainers used for oral drug delivery. The mechanism of the masking procedure with respect to the physicochemical characteristics of PDMS assured full coverage of appointed areas on a substrate. The temperature and time of the masking process were optimized and mathematically modelled. It was found that the self-aligning PDMS-based masking enabled a more precise and clean drug filling process and higher amount of an active compound loaded in microcontainers compared to a microfabricated Ni shadow mask. Finally, the PDMS masking method had the capacity to cover areas between microdevices, regardless of geometry, 
dimension, surface roughness or material. We recommend this method for various areas of research as well as commercial use in industry due to availability and low cost of PDMS and scalability of the technique.

Additional Supplementary Information may be found in the online version of this article.

\section{Declarations}

Funding This research was financially supported by the Danish National Research Foundation (DNRF122), Villum Fonden (Grant No. 9301) and the Novo Nordisk Foundation (NNF17OC0026910).

Conflict of interest The authors declare no conflicts of interest.

Availability of data and material Not applicable

Code availability Not applicable

\section{References}

Z. Abid, C. Gundlach, O. Durucan, C. von Halling Laier, L. H. Nielsen, A. Boisen, and S. S. Keller, Microelectron. Eng. 171, 20 (2017).

Z. Abid, S. Strindberg, M. M. Javed, C. Mazzoni, L. Vaut, L. H. Nielsen, C. Gundlach, R. S. Petersen, A. Müllertz, A. Boisen, and S. S. Keller, Lab Chip 19, 2905 (2019).

H. Becker and C. Gärtner, Anal. Bioanal. Chem. 390, 89 (2008).

J. Chen, M. Chu, K. Koulajian, X. Y. Wu, A. Giacca, and Y. Sun, Biomed. Microdevices 11, 1251 (2009).

X. Chen, N. Qu, H. Li, and Z. Xu, Appl. Surf. Sci. 343, 141 (2015).

X. Chen, N. Qu, H. Li, and D. Zhu, Adv. Mech. Eng. 6, 943092 (2014).

H. D. Chirra and T. A. Desai, Small 8, 3839 (2012).

A. J. Chung, Y. S. Huh, and D. Erickson, (n.d.).

C. G. Courcimault and M. G. Allen, in Nanotechnology (2004).

L. Dorogin and B. N. J. Persson, Soft Matter 14, 1142 (2018).

C. B. Fox, Y. Cao, C. L. Nemeth, H. D. Chirra, R. W. Chevalier, A. M. Xu, N. A. Melosh, and T. A. Desai, ACS Nano 10, 5873 (2016).

C. B. Fox, J. Kim, L. V. Le, C. L. Nemeth, H. D. Chirra, and T. A. Desai, J. Control. Release 219, 431 (2015).

C. B. Fox, C. L. Nemeth, R. W. Chevalier, J. Cantlon, D. B. Bogdanoff, J. C. Hsiao, and T. A. Desai, Bioeng. Transl. Med. 2, 9 (2017).

M. T. Ghannam and M. N. Esmail, Ind. Eng. Chem. Res. 37, 1335 (1998).

L. Jiang, X. Wang, and L. Chi, Small 7, 1309 (2011).

J. R. Jørgensen, M. L. Jepsen, L. H. Nielsen, M. Dufva, H. M. Nielsen, T. Rades, A. Boisen, and A. Müllertz, Eur. J. Pharm. Biopharm. 143, 98 (2019).

K. J. V. and S. G. V. K. Varadan, in Smart Mater. Syst. MEMS Des. Dev. Methodol. (John Wiley \& Sons, 2006), pp. 43-62.

S. S. Keller, F. G. Bosco, and A. Boisen, Microelectron. Eng. 110, 427 (2013). 
N. Kimura, M. Maeki, and M. Tokeshi, Microfluid. Pharm. Appl. 123 (2019).

Y. Luo, A. Cavus, M. C. Tamargo, J. Wan, and F. H. Pollak, J. Vac. Sci. Technol. B Microelectron. Nanom. Struct. 16, 1312 (1998).

P. Marizza, L. Pontoni, T. Rindzevicius, J. F. Alopaeus, K. Su, J. A. Zeitler, S. S. Keller, I. Kikic, M.

Moneghini, N. De Zordi, D. Solinas, A. Cortesi, and A. Boisen, J. Supercrit. Fluids 107, 145 (2016).

F. J. Martin and C. Grove, Biomed. Microdevices 3, 97 (2001).

C. Mazzoni, F. Tentor, S. S. Andersen, L. H. Nielsen, S. S. Keller, T. S. Alstrom, C. Gundlach, A. Mullertz, P. Marizza, and A. Boisen, J. Control. Release 268, 343 (2017).

L. H. Nielsen, S. S. Keller, and A. Boisen, Lab Chip 18, 2348 (2018).

L. H. Nielsen, S. S. Keller, K. C. Gordon, A. Boisen, T. Rades, and A. Müllertz, Eur. J. Pharm. Biopharm. 81, 418 (2012).

L. H. Nielsen, A. Melero, S. S. Keller, J. Jacobsen, T. Garrigues, T. Rades, A. Müllertz, and A. Boisen, Int. J. Pharm. 504, 98 (2016).

L. H. Nielsen, J. Nagstrup, S. Gordon, S. S. Keller, J. Østergaard, T. Rades, A. Müllertz, and A. Boisen, Biomed. Microdevices 17, 55 (2015).

C. Roh, J. Lee, and C. Kang, Materials (Basel). 9, 836 (2016).

A. Sayah, V. K. Parashar, A.-G. Pawlowski, and M. A. M. Gijs, Sensors Actuators A Phys. 125, 84 (2005).

T. Schallenberg, C. Schumacher, S. Gundel, and W. Faschinger, in Thin Solid Films (2002).

F. Schneider, J. Draheim, R. Kamberger, and U. Wallrabe, Sensors Actuators A 151, 95 (2009).

S. Selvarasah, S. H. Chao, C. L. Chen, S. Sridhar, A. Busnaina, A. Khademhosseini, and M. R. Dokmeci, Sensors Actuators, A Phys. 145-146, 306 (2008).

V. Silverio and S. Cardoso de Freitas, in Complex Fluid-Flows Microfluid. (Springer International Publishing, Cham, 2018), pp. 25-51.

T. W. Sowers, R. Sarkar, S. E. Prameela, E. Izadi, and J. Rajagopalan, Soft Matter 12, 5818 (2016).

S. K. Srivastava, F. Ajalloueian, and A. Boisen, Adv. Mater. 31, 1901573 (2019).

S. L. Tao and T. A. Desai, Adv. Drug Deliv. Rev. 55, 315 (2003).

S. L. Tao and T. A. Desai, Adv. Mater. 17, 1625 (2005).

L. Vaut, K. E. Jensen, G. Tosello, A. Khosla, H. Furukawa, and A. Boisen, J. Electrochem. Soc. 166, B3257 (2019).

L. Vaut, J. Juszczyk, K. Kamguyan, K. Jensen, G. Tosello, and A. Boisen, ACS Biomater Sci Eng (2020).

H. H. Winter and F. Chambon, J. Rheol. (N. Y. N. Y). 30, 367 (1986).

X. Wu, K. H. Gulden, M. Thomas, G. Wilson, J. Walker, G. H. Döhler, J. R. Whinnery, and J. S. Smith, J. Cryst. Growth (1993).

H. Zhang, J. K. Jackson, and M. Chiao, Adv. Funct. Mater. 27, 1703606 (2017).

A. Zosel, J. Adhes. 34, 201 (1991). 
A

1

2

3

4

5
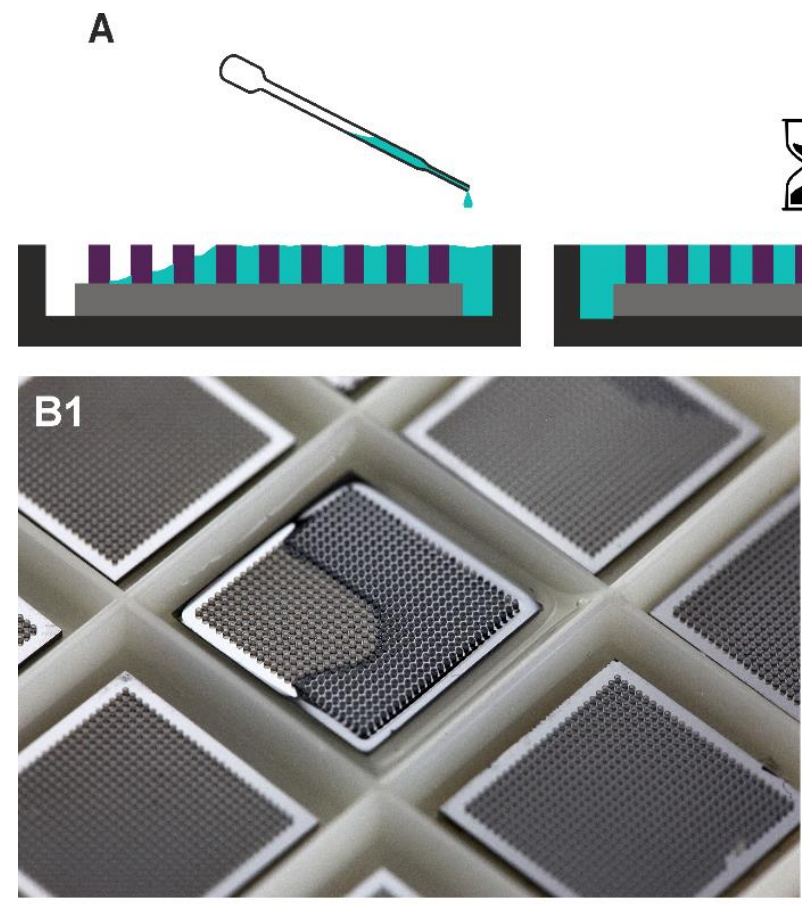

\section{B2}
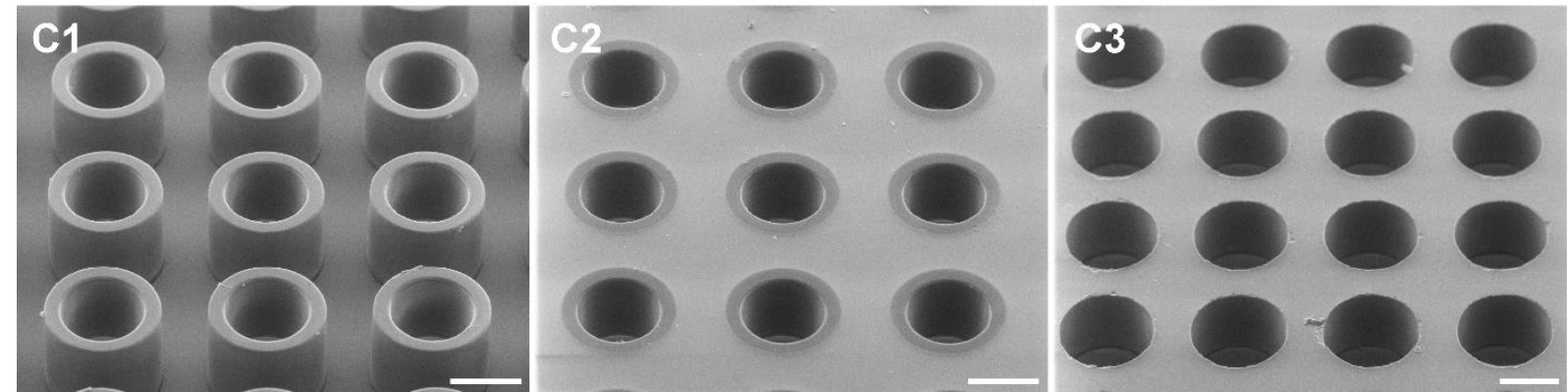

Figure 1. Fabrication of the PDMS mask, using microcontainers on a Si substrate. (A) Schematic illustration of the masking process. (B1) Capillary driven movement of PDMS between the microcontainers on a chip and (B2) peeling off the mask after crosslinking. (C1) SEM images of microcontainers before and (C2) after masking and (C3) the peeled off mask after crosslinking. Scale bar $=200 \mu \mathrm{m}$.

A

\begin{tabular}{|c|c|}
\hline Temperature (') & $\begin{array}{c}\text { Optimized crosslinking } \\
\text { time (min) }\end{array}$ \\
\hline 37 & $1165 \pm 23$ \\
\hline 50 & $130.8 \pm 9$ \\
\hline 70 & $26.2 \pm 4$ \\
\hline 90 & $7.4 \pm 0.3$ \\
\hline 100 & $4.4 \pm 0.9$ \\
\hline
\end{tabular}

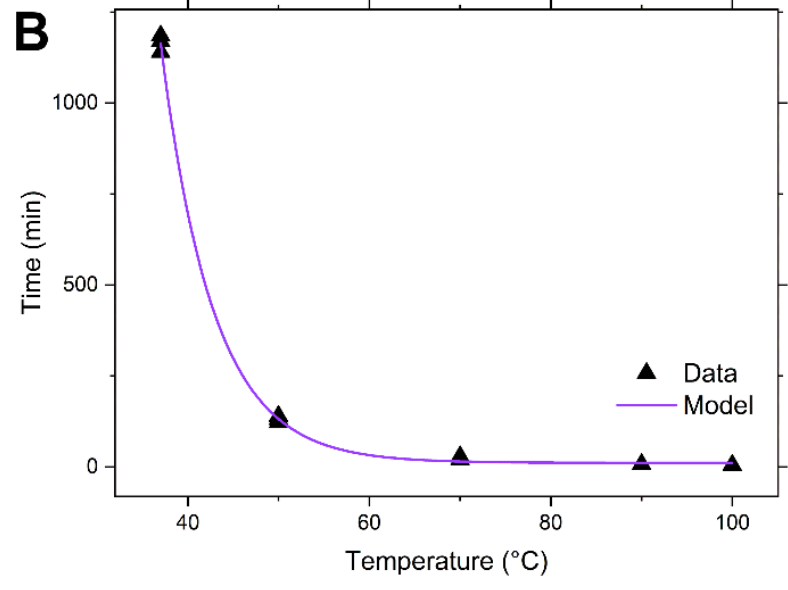

C

\begin{tabular}{|c|c|c|c|c|c|c|c|c|}
\hline & \multicolumn{2}{|c|}{$\mathbf{t}_{0}$} & \multicolumn{3}{c|}{} & \multicolumn{2}{c|}{ Statistics } \\
\hline & Value & $\mathrm{SD}^{*}$ & Value & $\mathrm{SD}$ & Value & $\mathrm{SD}$ & Chi-sq & $\mathrm{R}^{* * \mathrm{sq}^{* *}}$ \\
\hline $\mathbf{t}$ (min) & 11.1 & 4.2 & 714450.1 & 138018.2 & 5.8 & 0.2 & 151.958 & 0.999 \\
\hline
\end{tabular}


Figure 2. Temperature dependency of the duration of the PDMS masking process. (A) mask preparation time at various temperatures is presented as mean $(n=3) \pm$ standard deviation. (B) Non-linear curve fitting on mask fabrication time vs. temperature based on the model presented in equation (1). (C) Model parameters obtained by curve fitting presented in equation (1).

$* \mathrm{SD}=$ Standard deviation, $* *$ Chi-sq $=$ Reduced Chi-Square, $\mathrm{R}$-sq = Adjusted R-squared.
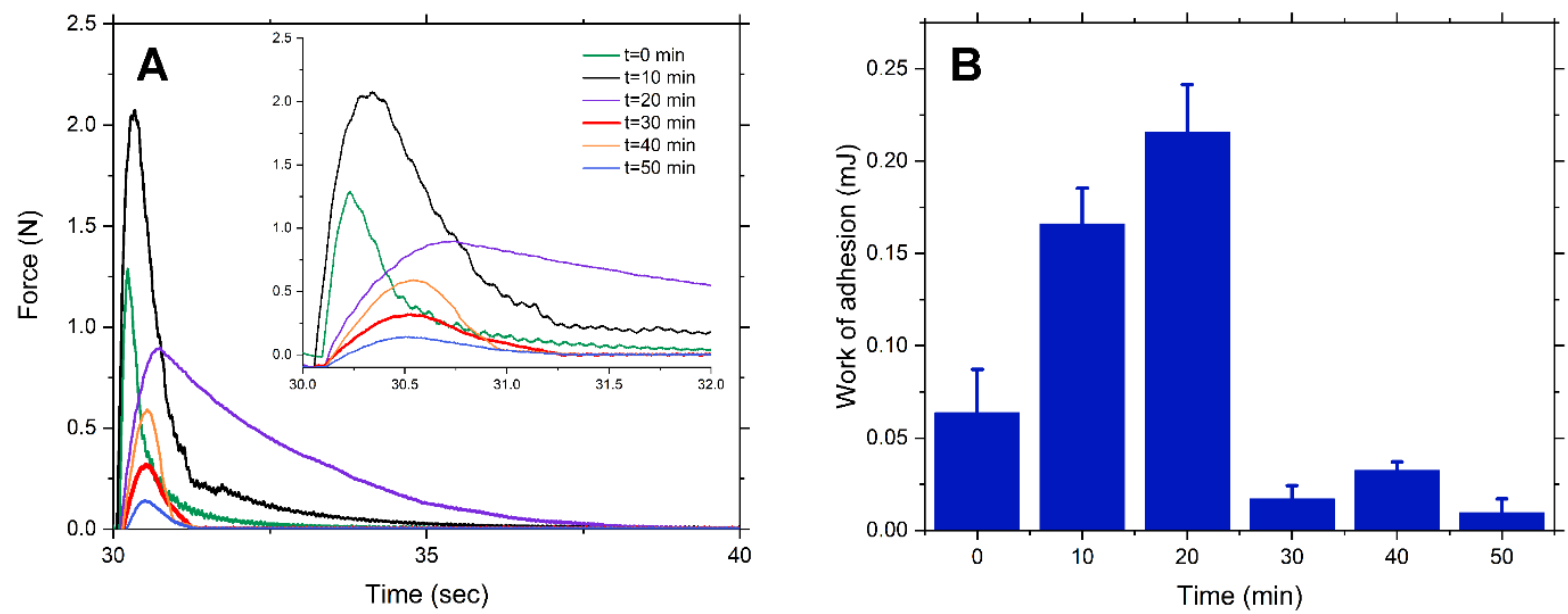

Figure 3. Physical and mechanical characterization of the masking process. (A) Representative detachment force curves after $30 \mathrm{sec}$ of contact; inset graph depicts the curves up to $32 \mathrm{sec}$. (B) Work of adhesion between the PDMS mask and a reference substrate at different time points at $70^{\circ} \mathrm{C}$ in plotted as mean $(n=3) \pm$ standard deviation. 

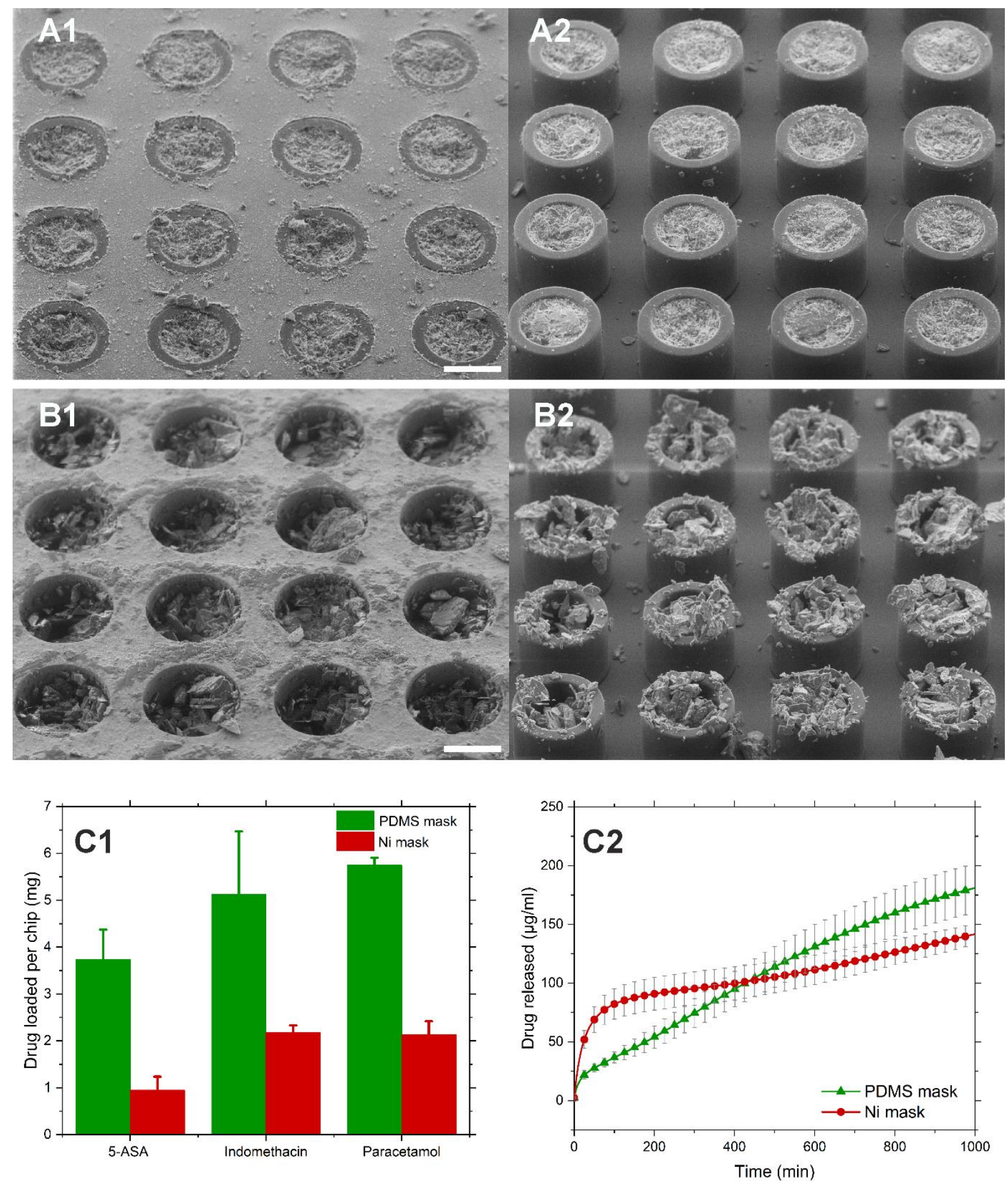

Figure 4. Comparison of powder drug loading using a PDMS and a Ni mask. SEM images of microcontainers loaded with indomethacin using PDMS (A1) before and (A2) after peeling off and the Ni mask (B1) before and (B2) after lifting. (C1) Comparing the loading capacity of microcontainers filled with 5-ASA, indomethacin and paracetamol, using the Ni and the PDMS mask. (C2) Release of indomethacin from microcontainers loaded with both techniques. Scale bar $=200 \mu \mathrm{m}$. Data in $\mathrm{C} 1$ and $\mathrm{C} 2$ is plotted as mean $(\mathrm{n}=3) \pm$ standard deviation. 

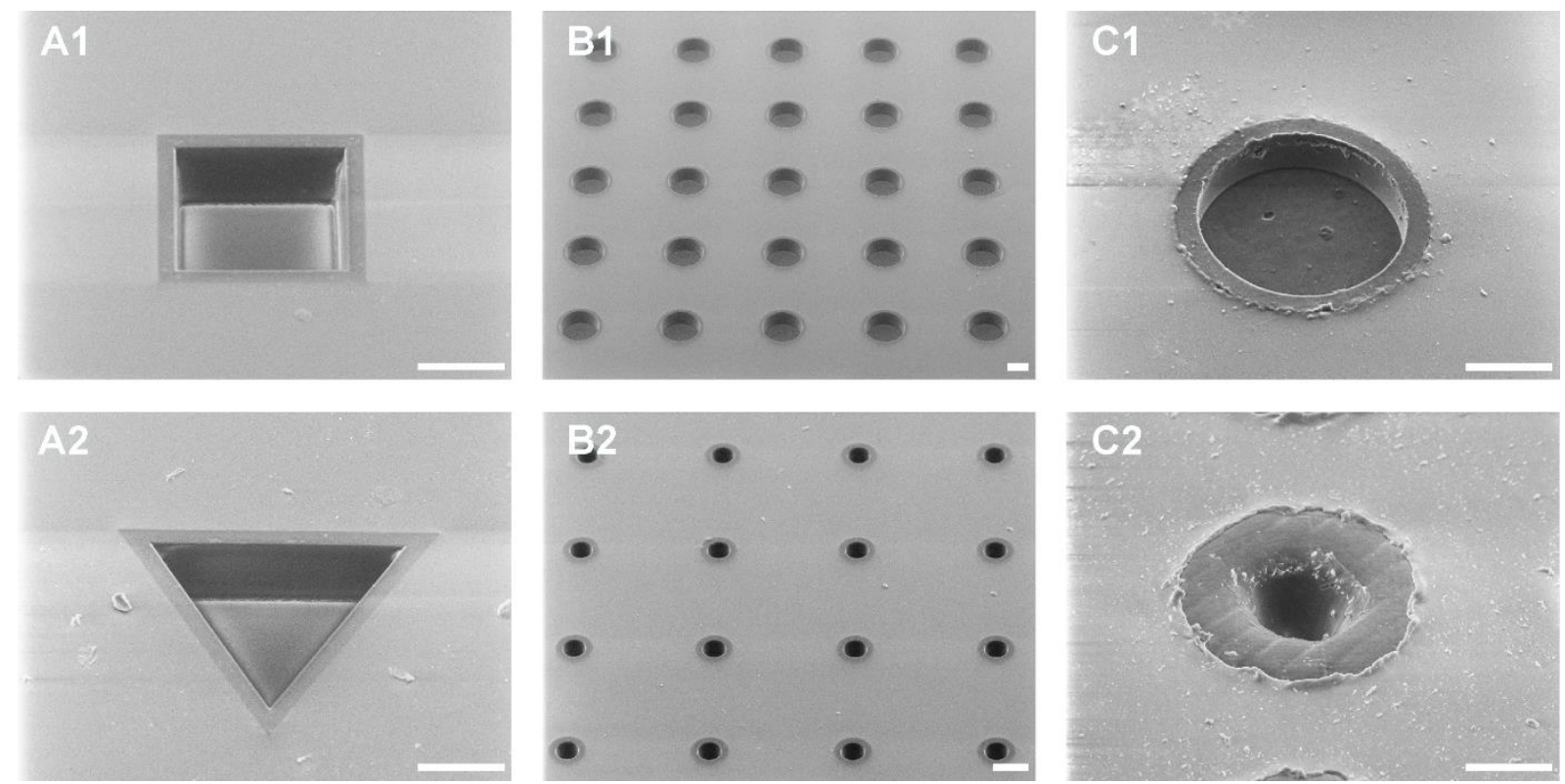

Figure 5. Versatility of the PDMS based technique, masking microdevices in the shape of (A1) a square and (A2) a triangle, size ranging from (B1) $260 \mu \mathrm{m}$ to (B2) $110 \mu \mathrm{m}$ in diameter and fabricated from (C1) PCL and (C2) HTM 140M V2. Scale bar $=100 \mu \mathrm{m}$.
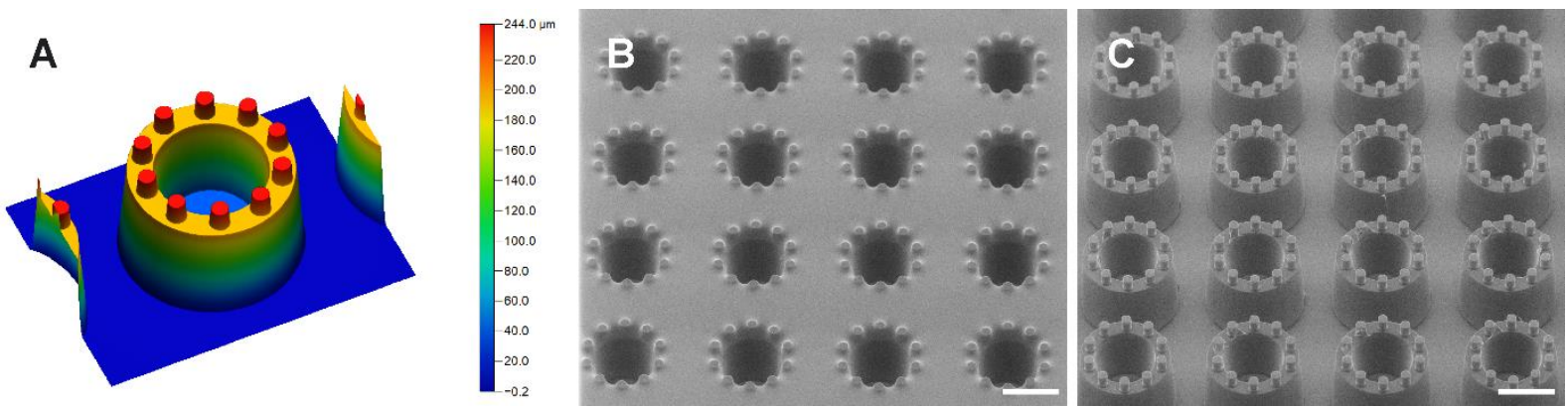

Figure 6. Compatibility of the method with complex topologies. (A) Optical profilometery image of microcontainers with 3D pillar-shaped structures on the sidewalls. SEM images of microcontainers with sidewall pillars before (B) and after (C) peeling off the PDMS mask. 


\title{
Development and Characterization of a PDMS-based Masking Method for
} Microfabricated Devices in Oral Drug Delivery

Khorshid Kamguyan ${ }^{1}$, Lasse Højlund Eklund Thamdrup ${ }^{1}$, Lukas Vaut ${ }^{1}$, Line Hagner Nielsen ${ }^{1}$, Kinga Zor $^{1}$, Anja Boisen ${ }^{1}$

\begin{abstract}
Acknowledgements The authors would like to acknowledge the Novo Nordisk Foundation (NNF17OC0026910) for funding the project MIMIO - Microstructures, microbiota and oral delivery and Danmarks Grundforskningsfond og Villum Fondens Center for Intelligent Drug Delivery and Sensing Using Microcontainers and Nanomechanics (IDUN) whose research is funded by the Danish National Research Foundation (DNRF122) and Villum Fonden (Grant No. 9301).

The authors would like to thank Zarmeena Abid from National Center for Nanofabrication and Characterization, Technical University of Denmark for supplying the PCL microcontainers for this project and Jesper Scheel from the Department of Health Technology, Technical University of Denmark for photography of the PDMS mask fabrication process.
\end{abstract}

\footnotetext{
${ }^{1}$ The Danish National Research Foundation and Villum Foundation's Center for Intelligent Drug Delivery and Sensing Using Microcontainers and Nanomechanics, Department of Health Technology, Technical University of Denmark, Kgs. Lyngby, 2800, Denmark
}

Correspondence to: Khorshid Kamguyan; E-mail: khokam @dtu.dk; Orcid ID: 0000-0003-1754-3434 
Supplementary Information may be found online on the webpage of the journal Biomedical Microdevices.

\section{S1 Fabrication of the Ni shadow mask}

A nickel shadow mask was made by electroplating on a predefined SU-8 scaffold. Starting out with a single-side polished Si substrate, tone inversion in the positive resist $\mathrm{AZ}^{\circledR} 5214 \mathrm{E}$ (Microchemicals GmbH, Ulm, Germany) was used for making a $1.5 \mu \mathrm{m}$ thick lift-off resist mask, consisting of circular dots and markers for alignment during laser cutting of the individual shadow masks. A seed layer of $10 \mathrm{~nm} \mathrm{Ti}$ and $100 \mathrm{~nm} \mathrm{Au}$ was then deposited using E-beam evaporation (Temescal FC-2000, Ferrotec, NH, USA) and the lift-off was concluded by immersion in microposit ${ }^{\mathrm{TM}}$ remover 1165 (Microchemicals $\mathrm{GmbH}$, Ulm, Germany) followed by immersion in isopropanol and DI water rinse. The approximately $420 \mu \mathrm{m}$ high SU-8 pillars were defined using a two-layer UV exposure scheme. After spin coating the first layer, a solvent bakeout at $55^{\circ} \mathrm{C}$ was performed for 10 hours and then maskless

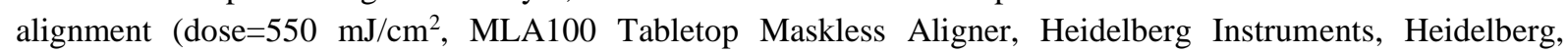
Germany) was employed for the first exposure. The exposed substrates were subject to a post-exposure bake (PEB) for 4 hours at $55^{\circ} \mathrm{C}$ before spin coating the second layer. After a 15 -hour solvent bakeout at $55^{\circ} \mathrm{C}$, the second UV exposure was conducted $(\mathrm{dose}=650 \mathrm{~mJ} / \mathrm{cm} 2)$ and a 15 -hour $\mathrm{PEB}$ at $55^{\circ} \mathrm{C}$ was carried out before developing the structures by $2 \times 20 \mathrm{~min}$ immersion in mr-Dev 600 (micro resist technology GmbH, Germany). The developed substrates were then flushed with isopropanol and dried using nitrogen. The approximately $280 \mu \mathrm{m}$ thick $\mathrm{Ni}$ shadow masks where then electroplated (Technotrans microform.200, Germany) using a maximum current of 3.5 A, a setpoint charge of 14.1 Ah, a pH of 3.7 and a total plating time of 5 hours. After electroplating, the Si substrate was etched away in 7 hours using a $50 \mathrm{vol} \% \mathrm{KOH}$ bath at $85^{\circ} \mathrm{C}$. The SU-8 scaffold was then removed in a Piranha solution $\left(4: 1 \mathrm{H}_{2} \mathrm{SO}_{4}: \mathrm{H}_{2} \mathrm{O}_{2}\right)$ by immersion for $15 \mathrm{~min}$. For cutting out the individual $12.8 \times 12.8 \mathrm{~mm}^{2}$ shadow masks, a laser micromachining tool (microSTRUCT vario, 3D-Micromac AG, Germany) operating at $1064 \mathrm{~nm}$ was utilized. The system was equipped with a $50 \mathrm{~W}$ picosecond laser (FUEGO, Time Bandwidth) and through-cuts were obtained using 400 iterations. The laser cut shadow masks were then finally cleaned using sonication in DI water with Triton X-100 followed by sonication for $20 \mathrm{~min}$ in isopropanol. The resulting shadow mask is presented in Figure S1.
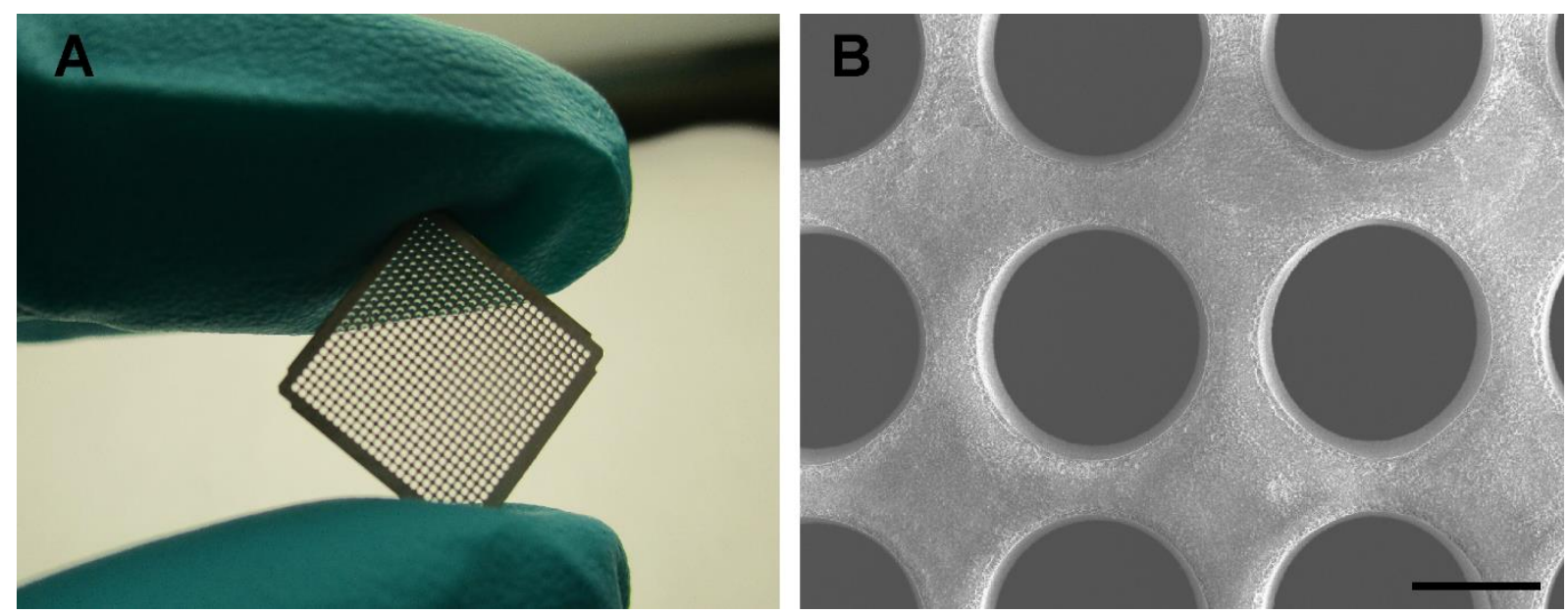

Figure S1. (A) Ni shadow mask fabricated through an electroplating procedure on a SU-8 scaffold. (B) SEM image of the shadow mask after fabrication. Scale bar $=200 \mu \mathrm{m}$. 


\section{S2 Fabrication and Characterization of the microcontainers}

Within the framework of the current study, microcontainers, having different topologies, were produced by conventional UV-lithography in the well-known epoxy-based negative photoresist SU-8. The large cylindrical microcontainers were made using a conventional aligner (Süss Mask Aligner MA6 (equipped with i-line notch filter), SUSS MicroTec, Germany), whereas cylindrical microcontainers with micro-pillars on the sidewalls and also the multitude of other containers having round, square or triangular geometries were made using a maskless aligner (MLA100 Tabletop Maskless Aligner, Heidelberg Instruments, Germany). The overall process flow was composed of spin coating a $35 \mu \mathrm{m}$ thick bottom layer (SU-8 2035) on a single-side polished 4" Si substrate (thickness of $500 \mu \mathrm{m}$ ), which was then subject to a solvent bakeout at $50^{\circ} \mathrm{C}$ (ramping $=2^{\circ} \mathrm{C} / \mathrm{min}$. This ramping was used for all hotplate processes) for 2 hours. Next followed a UV exposure (doses in excess of $250 \mathrm{~mJ} / \mathrm{cm}^{2} \mathrm{was}$ used) and a post exposure bake (PEB) at $50^{\circ} \mathrm{C}$ lasting 6 hours. For defining the sidewalls of the drug vessels, a thicker layer of SU-8 (SU-8 2075) was spin coated in order to obtain a thickness of approximately $220 \mu \mathrm{m}$ for the cylindrical microcontainers, $160-170 \mu \mathrm{m}$ for the microcontainers with sidewall pillars and $85 \mu \mathrm{m}$ for the cornucopia microdevices having round, square and triangular geometries. Again, a solvent bakeout was performed at $50^{\circ} \mathrm{C}$ and the second layer was exposed (doses ranging from $300-500 \mathrm{~mJ} / \mathrm{cm}^{2}$ ) and baked at $50^{\circ} \mathrm{C}$. For the microcontainers with sidewall pillars, a final layer of SU-8 (SU-8 2035) was spin coated in order to define the micro-pillars having a height of approximately $40 \mu \mathrm{m}$. The same hotplate temperature, as previously stated, was used for both the solvent bakeout and the PEB. Development of the exposed substrates was done using $2 \times 20$ minutes immersion in mr-Dev 600 (micro resist technology GmbH, Germany) and flushing the developed structures with copious amounts of isopropanol before drying the substrates with pure nitrogen. Topology characterization was done using conventional bright-field microscopy (Nikon Eclipse L200, Nikon Metrology, Japan) and vertical scanning interferometry (PLu Neox 3D Optical Profiler, Sensofar Metrology, Spain).

Microcontainers used for demonstrating the PDMS masking process and drug loading were inspected with vertical scanning interferometry to investigate the exact dimensions (Figure S2). The microcontainers with different shapes and sizes were characterized using optical microscopy as revealed in Figure S3.

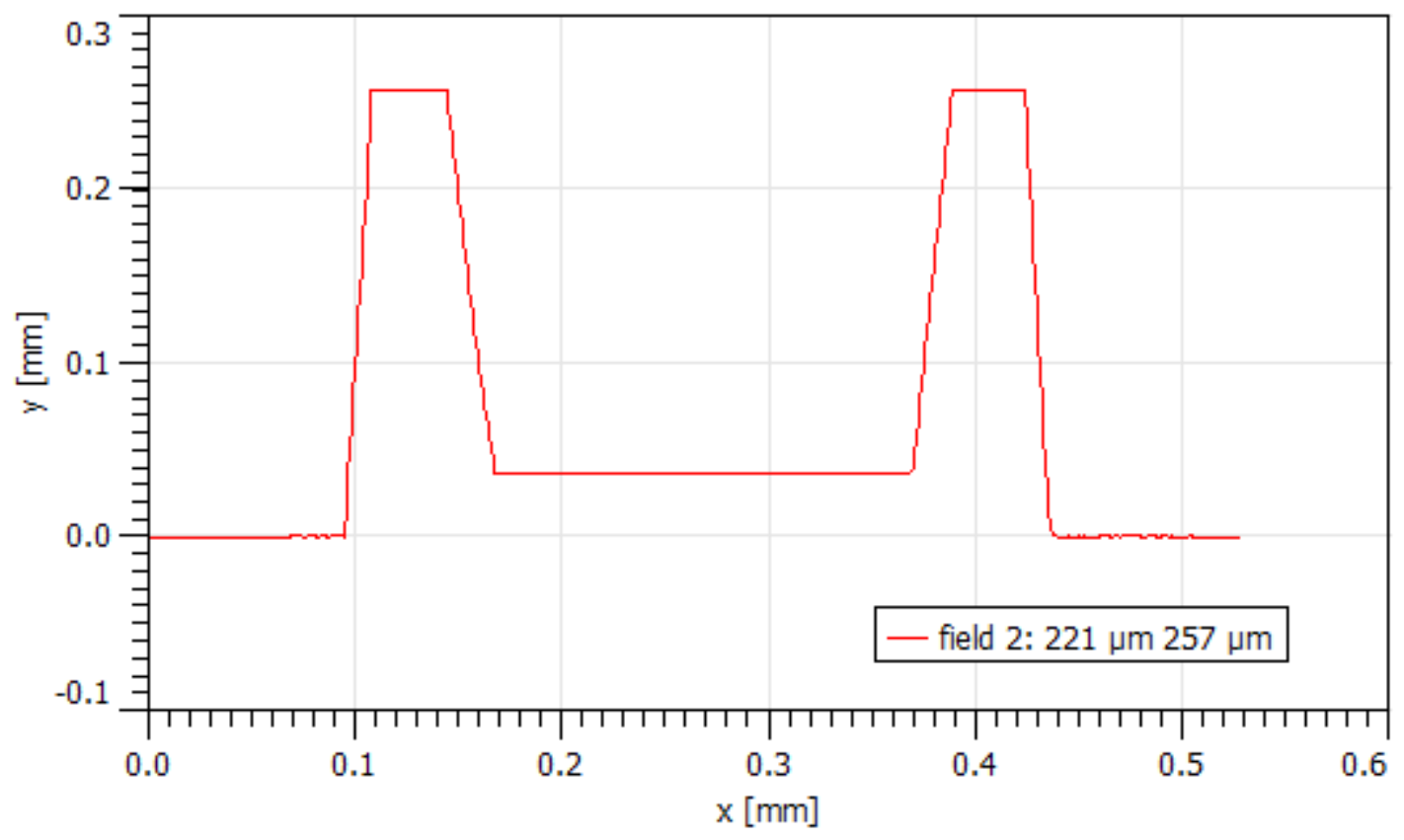

Figure S2. Optical profilometery image of an SU-8 microcontainer with an average outer height of $256 \mu \mathrm{m}$ and an average outer diameter of $325 \mu \mathrm{m}$. 


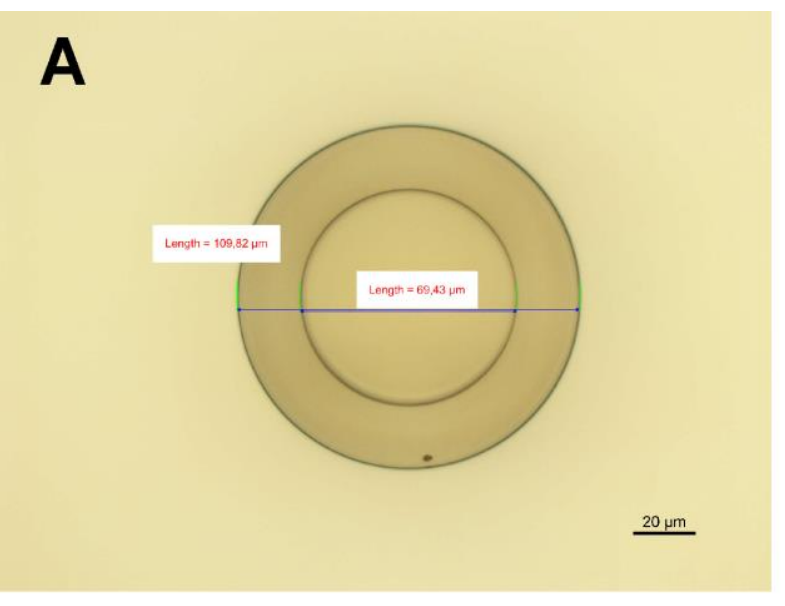

\section{B}

C

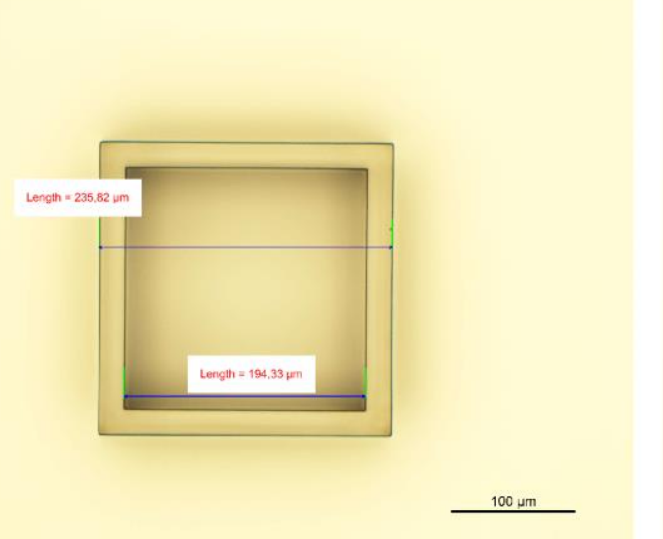

D
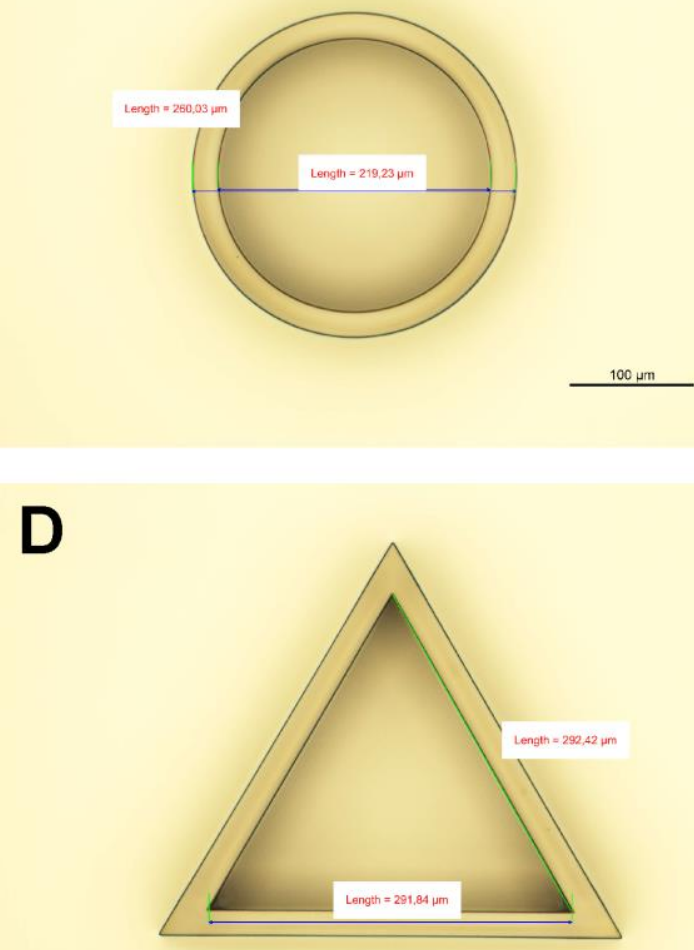

Figure S3. Optical microscopy images of microcontainers with different shapes and sizes; (A) small and (B) large circles, (C) Squares and (D) triangles.

S3. Characterization of loading 5-ASA and paracetamol in microcontainers using PDMS and Ni masks 

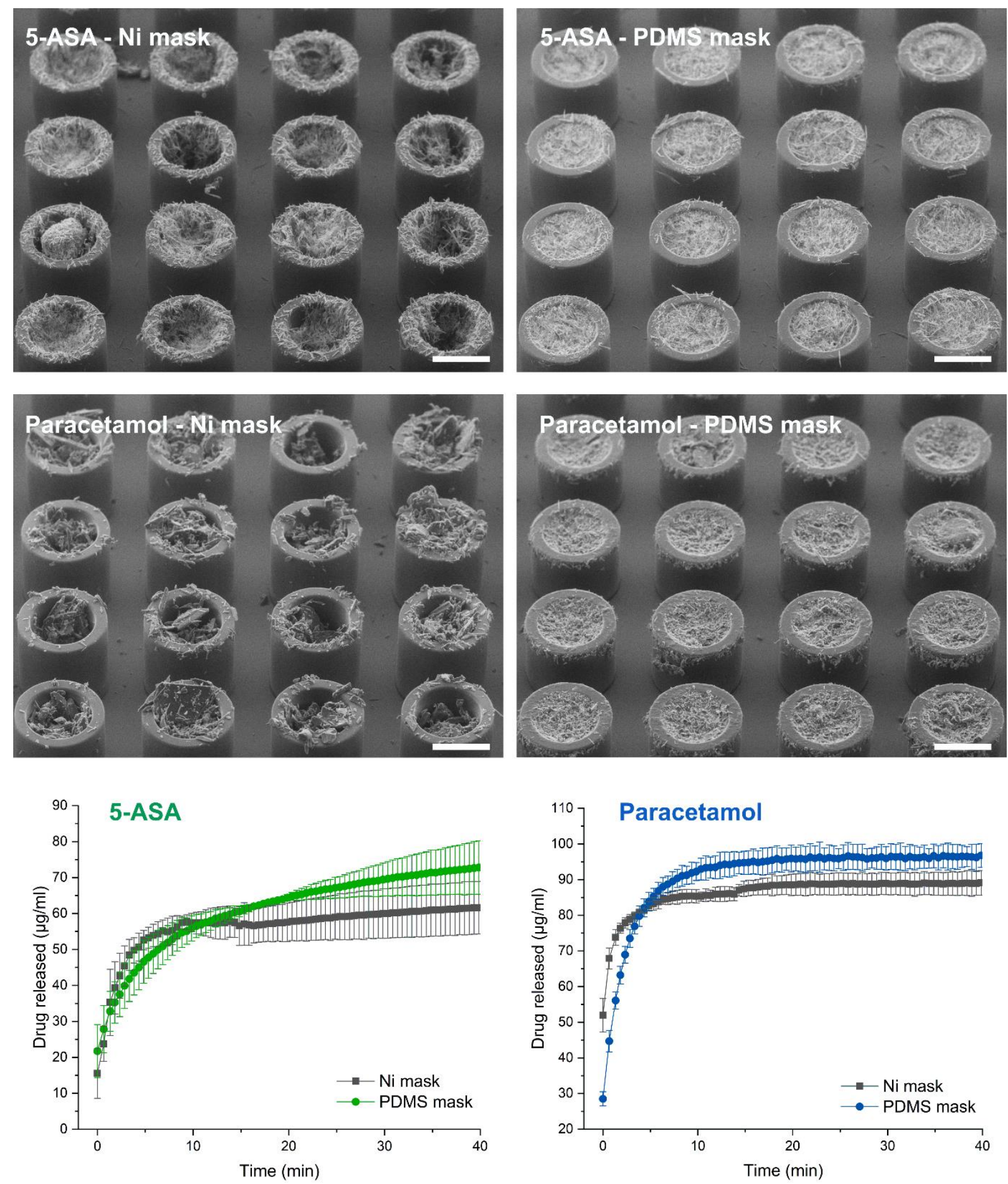

Figure S4. SEM images of microcontainers loaded using PDMS and Ni masks filled with (first row) 5-ASA and (second row) paracetamol. (Third row) release of 5-ASA and paracetamol from microcontainers loaded using the PDMS and Ni mask in phosphate buffer saline. $n=3 \pm$ standard deviation. 